\title{
Innovation and Practice of Electronic Circuits
}

\author{
Hua Fan, Weijian Chen, Jin Zhang, Yulan Li, Xingning Ye, and Quanyuan Feng
}

\begin{abstract}
The vigorous development of electronic technology imposes new demands on how to cultivate talents for the electronics industry, traditional mode of education has failed to meet the new requirements, in the new century, education has become the focus of the reform, and cross, penetration and integration between basic courses are the key to improve the quality of teaching and the overall quality of students. University of Electronic Science and Technology of China (UESTC) combines circuit analysis and fundamentals of analog circuits as one course electronic circuit, which is one of the most important projects of curriculum reform in 2015, and the curriculum reform follows the principles of strengthening the foundation, updating the structure, penetrating the interdisciplinary and simplifying the courses. According to teaching experience and the actual situation of our university, this paper discusses the principles and ideas of reforms related to the electronic circuit: the results show that the teaching can broaden the knowledge and vision of students, more comprehensive and accurate understanding about analog circuits of students can be achieved, a solid foundation can be formed for the study of follow-up courses, as a result, the students can better adapt to the requirements of learning and challenge of the new era.
\end{abstract}

Index Terms-Analog-to-digital converter, electronic circuits, feedback, operational amplifier, regulator.

\section{INTRODUCTION}

With the trend of new global information technology in the 21st century, cloud computing, big data, Internet of Things, robots, mobile communications, virtual reality and other areas of vigorous develops in an unbelievable speed. So far, no subject can be as colorful and ever-changing as the field of information technology. Mankind has entered the era of information technology, represented by hi-tech innovation.

Manuscript received December 3, 2017; revised July 12, 2018. This work was supported in part by the Natural Science Foundation of China (NSFC) under Grant 61771111 and 61401066, supported by China Postdoctoral Science Foundation under grant 2017M612940, Special Foundation of Sichuan Provincial Postdoctoral Science Foundation, as well as supported by Teaching and assessment methods reform demonstration course "Foundations of Analog Circuit" course construction (2017XJYJ-ZD06); electronic circuit (circuit analysis and analog circuit) course integrated teaching (2015XJYZC004); Communication Bachelor comprehensive reform of professional education (2015XJYZD 001); Professional recognition as an opportunity to continuously improve the training of communication engineering professionals (2015XJYZD 002)] ; The work of Quanyuan Feng was supported by the National Natural Science Foundation of China (NSFC) under Grant 61531016, supported by the project of Science and Technology Support Program of Sichuan Province under Grant 2015GZ0103, and in part by the Sichuan Provincial Science and Technology Important Projects under Grant 2017GZ0110.

Hua Fan, Weijian Chen, Jin Zhang, Yulan Li, and Xingning Ye are with University of Electronic Science and Technology of China, Chengdu, China (e-mail: fanhua7531@163.com).

Quanyuan Feng is with Southwest Jiaotong University, Chengdu, China (e-mail: fengquanyuan@163.com).
The development of information technology is very rapid. Moore's Law (the performance is doubled every 18 months and the prices are down by half), the Melkov's law (the network value is equal to the square of the network node) and the new Moore's Law (the Internet bandwidth will double the capacity every 9 months, but the cost is also reduced by half) And so on, as some economists have said: "The expansion and infiltration of information technology across the whole society is tantamount to the second industrial revolution, even more profound than the impact of the first industrial revolution."

February 18, 2017, the Ministry of Education held a seminar on the development of higher engineering education strategy in Fudan University, reached a "new engineering" construction Fudan consensus. New technologies, new formats, new industries, new models are the characteristics of the new economy booming, and there has been an urgent need to cultivate a large number of innovative new engineering talents. Compared with the traditional engineering education, "new engineering" talent should possess the basis of knowledge, professional ability, high comprehensive quality, with international competitiveness, and to adapt to the era of pan-information, dare to cross border and be capable of cross-border innovation. What's more, the most important work is to develop students' core competencies - the ability to learn new things quickly, and students need to learn in knowledge cross and integration.

The transformation of the times prompted us to carry out teaching reform, reconstruct some core knowledge in curriculum construction, upgrade the old knowledge, combine new technology with the teaching content and focus on learning ability and engineering thinking to improve the teaching design. University of Electronic Science and Technology began to explore in 2013 to study the ability to cultivate as the core, to industry development needs as the standard, the implementation of the curriculum system optimization and integration, has made some good results. This article will detail the "electronic circuit foundation" course through teaching reform.

\section{AN ANALYSIS OF THE PROBLEMS IN TRADITIONAL TEACHING}

\section{A. Two Independent Courses Are not Conducive to Master}

Traditional teaching has the "circuit analysis" and "fundamentals of analog circuits" as two independent courses, "circuit analysis" course for 64 hours, opened in the second semester of new students, and "fundamentals of analog circuits" course for 64 hours, opened in the first semester of sophomore, that is, "circuit analysis" functions as the prerequisite course of the "fundamentals of analog circuits". 
Two courses originally belong to the relevant knowledge systems, independent teaching are not conducive to mastery. For example, the Kilchoff law (KCL, KVL), which is described in Chapter 1 of the "circuit analysis", is the basic rule for the voltage and current in all circuits and is the basis for the analysis and calculation of more complex circuits. In the "fundamentals of analog circuits", these basic rules can be used for the analysis of DC circuits, can also be used for the analysis of $\mathrm{AC}$ circuits and for the analysis of nonlinear circuits; "external power method" in Chapter II of "circuit analysis" is always used to simplify the circuit, by applying an external voltage source or external current source to solve the current or voltage to finally get the equivalent input, output resistance of amplifier circuit, etc. "Circuit with controlled source analysis" in chapter III in "circuit analysis" and "Superposition theorem, the Thevenin theorem and the Norton theorem" in the fourth chapter in "circuit analysis" are commonly used to simplify and analyze the transistor amplifier circuit, FET amplifier circuit and multi-stage amplifier circuit in "fundamentals of analog circuits". The shortcomings of the two courses, "circuit analysis" and "fundamentals of analog circuits", which are taught in two separate semesters, lie to the fact that most of the students have become unfamiliar about the critical knowledge of "circuit analysis" when they start learning "fundamentals of analog circuits", so that students may face some difficulties in the cognition and application of relevant knowledge.

\section{B. Out of Touch between Theoretical Teaching and Experimental Practice}

Two courses are divided into different semesters, making the teaching process hard to combine theoretical analysis, simulation design, experimental verification of electronic circuit analysis and design methods, along with theory and practice being out of touch. For example, the traditional circuit analysis courses, theoretical teaching only starts teaching from the circuit model (graphical mathematical model), without taking the actual problems (such as the type of resistance, power and tolerance, etc.) into consideration, once the experimental and theoretical analysis is not complete anastomosis, the students will find it difficult to understand.

Therefore, our school integrates "fundamentals of analog circuits" and "circuit analysis" into one course- "electronic circuit", 96 hours, 6 credits, opened in the second semester of freshman, the key knowledge point of "circuit analysis" are inserted into the "fundamentals of analog circuits". The reform of the "electronic circuit" course embodies the organic integration of teaching content in-depth and step by step, from the abstract components to the circuit model analysis, and then to the electronic circuit analysis and design, from passive devices to active devices, from linear to non-linear, from the actual circuit to the circuit model, from the unit circuit to the functional circuit, from the circuit analysis to the circuit design, has eliminated the barrier of "circuit analysis" and "fundamentals of analog circuits". This is conducive to the mastery of knowledge. This is the only discipline which owns six credits of the professional basic course in the University of Electronic Science and Technology, the teaching reform project - integrated teaching of electronic circuit ("circuit analysis" and "fundamentals of analog circuits")
(2015XJYZC004) has been included as one of the major teaching reform projects in 2015 .

\section{INTEGRATION PROGRAM OF "CIRCUIT ANALYSIS" "FundAMENTALS OF ANALOG CIRCUITS"}

\section{A. Scheme of Integration Program}

The integration of the two courses of "circuit analysis" and "fundamentals of analog circuits" is not a simple superposition. The traditional "circuit analysis" course adopts national key excellent teaching materials, which belongs to general high education "Ninth Five-Year", edited by Qiu Guanyuan, including two parts: the first Part of circuit steady state analysis, including the circuit model and circuit law, the equivalent conversion of the resistance circuit, the general analysis of the resistance circuit, the circuit theorem, including the operational amplifier resistance circuit, phasor method, sinusoidal steady-state circuit analysis; The second part of the circuit dynamic analysis, including the first order circuit, the second order circuit, the Laplace transform, the network parameter, the circuit equation matrix form, two-port network, non-linear circuit [1], [2]. After the integration of the two courses, "Circuit analysis" and "fundamentals of analog circuits" [3]-[7], the content of the circuit analysis has been reduced by half, which is due to the rapid development of electronic circuit technology, computer-aided analysis of the circuit has become widely adopted scientific research methods, electronic design automation (EDA) and the rapid development of various circuit simulation software greatly simplifies the past complex circuit analysis and calculation, therefore, teaching should strengthen the "circuit computer-aided analysis", so that students can have basic knowledge of the large-scale circuit computer aided analysis of the methods and processes, and establish "scientific computing" concept, the analysis of the internal principles of the module should not be too detailed and the complexity of the calculation of circuit should be reduced, but the classical theoretical knowledge of "circuit analysis" and necessary knowledge of transition from "circuit analysis" to "fundamentals of analog circuits" need to be refined, in addition, timely and accurate summary are imperative. The integration program of the two courses is as shown below:

Chapter 1: Circuit and circuit model, including

- Understand the composition of the circuit, the role of the basic variables of circuit analysis

- Maintain resistance, independent power supply, inductors, capacitors and other components of the VCR, understand the resistance, power, inductors, capacitors and other basic components and models

- Master Kirchhoff's law and its application

- Master the controlled power supply VCR, understand the diode, transistor, FET and other semiconductor devices and their models

\section{Chapter 2: Circuit analysis method, including}

- Master the resistor divider circuit and shunt circuit

- Master the node analysis method

- Master the superposition theorem and its application

- Understand single-port network and network equivalent

- Master Thevenin - Norton theorem and its application 


\section{Chapter 3: Semiconductor device, including}

- Understand the basic knowledge of the semiconductor

- Understand the diode, regulator and other semiconductor devices and their models

- Master the controlled power supply VCR, understand the transistor, FET and other semiconductor devices and them Models

Chapter 4: Single transistor amplifier circuit, including

- Understand the role of amplification circuit, composition, amplification circuit performance indicators and amplification circuit model

- Master the transistor common emitter amplifier circuit, understand the static operating point stability and DC negative feedback technology

- Master the transistor common collector amplifier circuit and the common base amplifier circuit

- Master the field effect transistor common source amplifier circuit and the total leakage amplifier circuit

Chapter 5: Sinusoidal steady state circuit, including

- Understand the phase of the sinusoidal steady-state circuit with the amount of sinusoidal representation

- Master the form of Kirchhoff's law

- Control the resistance, independent power, controlled power, inductance, capacitance and other components VCR phasor form, impedance and admittance

- Master the phasor analysis of sinusoidal steady-state circuits

- Understand the resonance in the sinusoidal steady-state circuit

Chapter 6: The frequency response of the amplifier circuit, including

- Understand the concept of frequency characteristics

- Understand the transistor high frequency model and the field effect transistor high frequency model

- Master the frequency characteristics of a single tube amplifier circuit

Chapter 7: Multistage Amplifier and Integrated Operational Amplifier, including

- Master the multi-stage amplifier circuit

- Master the differential amplifier circuit

- Master the integrated current source and integrated differential amplifier circuit

- Master the complementary output circuit

- Understand the integrated circuit of the operational amplifier

- Understand the performance indicators and models of integrated operational amplifiers

Chapter 8: Negative feedback amplifier circuit, including

- Understand the concept of feedback

- Understand the four configurations of the negative feedback amplifier circuit

- Master the depth of the negative feedback amplifier circuit magnification

- The effect of negative feedback on the other performance of the amplifier circuit

Chapter 9: Operation circuit and filter circuit, including

- The effect of negative feedback on the other properties of the amplifier circuit

- Understand integral, differential operation circuit, logarithmic, exponential operation circuit, multiplication, division operation circuit

- Understand low-pass, high-pass, band-pass and band-stop filter circuit

\section{B. The Key Points of Integrated Teaching of "Circuit Analysis" and "Fundamentals of Analog Circuits"}

The "circuit analysis" course should be the basis of the "fundamentals of analog circuits" course, two chapters "circuit and circuit model" and "circuit analysis method" enable students to master the basic knowledge of electronic circuits, transfer the complex engineering problems into circuit models, then deduce and calculate accurately according to the established model. After studying the "circuit and circuit model" and "circuit analysis method" in "circuit analysis", we can start to study the "semiconductor device" and "single transistor amplifier circuit" in the "fundamentals of analog circuits", because once the students understand the basic concept of the circuit model and the superposition theorem, the Thevenin theorem and the Norton theorem, they can use these theorems to analyze the amplifying circuit with the dual-port equivalent microcircuit. For example, when the amplifier is analyzed separately, the amplifier can be regarded as a passive dual-port network. Considering the signal source, the amplifier should be regarded as the load of the signal source. The amplifier and the load should be considered as a passive single-port network, as shown in Fig. 1. The network is equivalent to the resistance $\mathrm{Ri}$, that is, the input resistance of the amplifier $\mathrm{Ri}$ is the load resistance of the signal source. From the load side, the signal source and the amplifier circuit are equivalent to the one port net containing source, as shown in Fig. 3. For the analysis of the one port net containing source, the equivalent circuit can be derived according to Thevenin theorem or the Norton theorem, which is equivalent to the open circuit voltage source $\mathrm{U}_{\mathrm{OC}}$ and output resistor Ro series or short-circuit current source $\mathrm{I}_{\mathrm{SC}}$ and output resistance Ro in parallel. In the "fundamentals of analog circuits" mentioned in the analysis of diode, transistor and FET, the superposition theorem need to be used, you need to specifically emphasize that only the transistor with a small signal model to do the linearization of the processing before using the superposition theorem, otherwise, non-linear circuit cannot be solved by using the superposition theorem.

Above two chapters belong to contents of "fundamentals of analog circuits", the fifth chapter re-enters into the "circuit analysis", sinusoidal steady-state circuit, which is basics for the sixth chapter "frequency response of amplifier circuit", for the amplifier circuit, we need to study the amplitude and frequency characteristics and phase frequency characteristics of amplification circuit with the sine input, the sinusoidal signal is time-varying, its amplitude and phase change with time, for the time-varying signal, phasor method is usually used, phasor is used in electrical engineering to represent the amplitude and phase of the sine vector, when the frequency is constant, the phasor is the only character of sine, therefore, the amplifier circuit is characterized by the phasor analysis of the sinusoidal steady-state circuit. As a result, before learning the frequency response of the amplifier circuit, it is necessary to talk about the "sinusoidal steady-state circuit" so that the students can be flexible using the phasor method to analyze the frequency characteristics of the amplifier circuit, and have 
a deep understanding that the gain of amplifier is a function of the signal frequency, as the input signal frequency is low or high to a certain extent, the gain will drop and phase will shift.

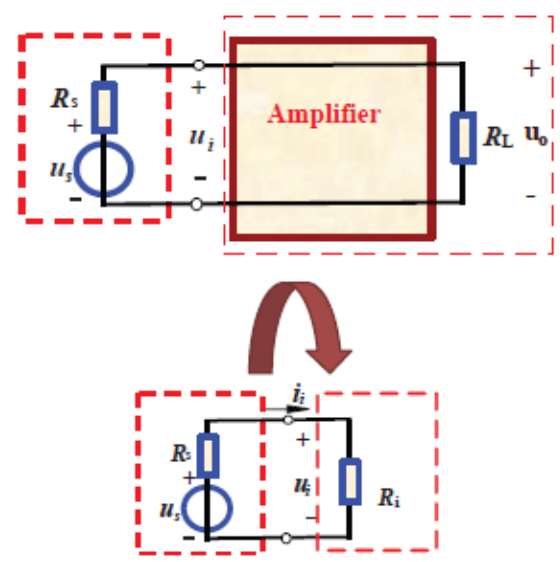

Fig. 1. The amplifier and the load consist passive single-port network.

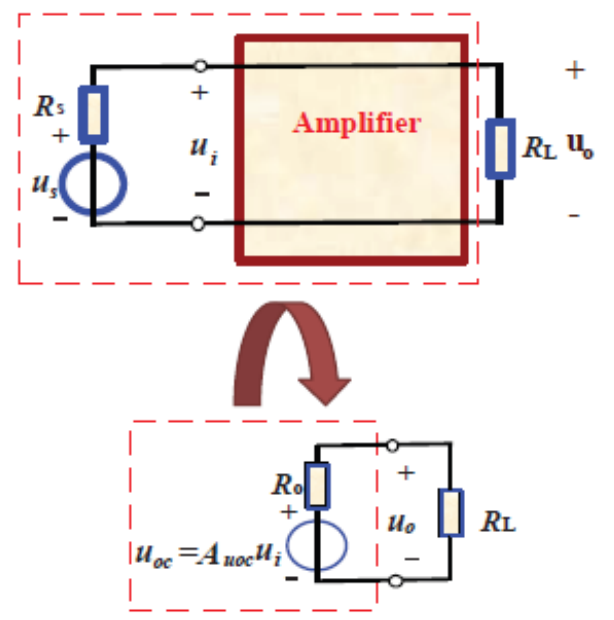

Fig. 2. The signal source and the amplifier circuit consist one port net containing source.

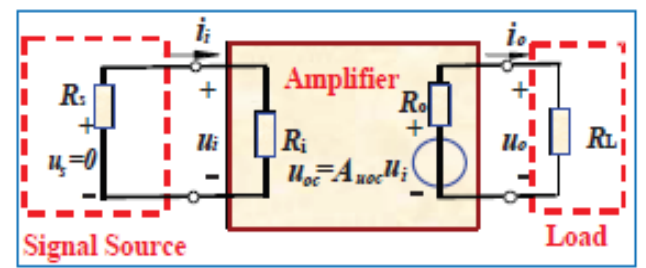

Fig. 3. Model derivation of amplifier of "fundamentals of analog circuits" through "circuit analysis".

The contents of the last three chapters of the "electronic circuit" belong to the "fundamentals of analog circuits", in which the feedback part is key and difficult, feedback and op amp are the essences of analog circuits, most of the op amps in electronic systems are used in the conditions of closed loop negative feedback, only to master the type of feedback can students understand the impact of negative feedback on the performance of the amplifier circuit, however, the type of feedback is not easy for students to learn, textbooks only gives method that students are hard to understand, we need to help students with summary of knowledge that textbooks do not include or not easy to understand.

\section{ACHIEVEMENT OF TEACHING REFORM}

In May 2016, the University of Electronic Science and
Technology held a seminar on teaching reform of electronic information courses in colleges and universities, which attracted more than 280 teachers and experts from 95 colleges and universities, including Tsinghua University, Southeast University, Shanghai Jiaotong University, Zhejiang University, Xi'an University of Electronic Science and Technology, Beijing University of Posts and Telecommunications, National University of Defense Technology, etc. We have made presentation with theme of "Integration and construction progress of circuit courses", which led to heated discussion, what is more, we exchange experience in the seminars held by other electronic information basic courses series of different universities, every year, other institutes come to University of Electronic Science and Technology to study and exchange learning progress, the effect of curriculum reform of University of Electronic Science and Technology is highly recognized.

The "electronic circuit" course taught by first author Hua Fan achieves more than 90 points from students' evaluation every semester. And on behalf of the University of Electronic Science and Technology, in August 25, 2016, Hua Fan participated in the young teachers teaching competition organized by the Ministry of Education, in the competition, with the new way of teaching which combines the knowledge of the "circuit analysis" with the "fundamentals of analog circuits" flexibly, Fan won the second prize in the southwest of China and presented the judges a refreshing feeling.

The second author Chen Weijian was awarded as "the most popular teacher" of the University of Electronic Science and Technology in 2012, 2016, and 2017, which is the highest honor that the University of Electronic Science and Technology gives teachers. As the chief professor of the course, he led the course group to carry out a large number of teaching reforms and research.

\section{CONCLUSION}

In order to deepen the reform of engineering education in colleges and universities, the University of Electronic Science and Technology launched a special teaching reform - the teaching of electronic circuit courses. Teaching reform based on breaking the original segmented teaching model, to achieve the curriculum knowledge system within the penetration and smooth transition, to promote the organic integration of curriculum content [8], to cultivate students' innovative thinking and engineering practice ability in addition, this helps students to solve complex problems and improve self-learning and lifelong learning ability.

\section{ACKNOWLEDGMENTS}

The work of Hua Fan was supported by the National Natural Science Foundation of China (NSFC) under Grant 61771111 and 61401066, supported by China Postdoctoral Science Foundation under grant 2017M612940 and Special Foundation of Sichuan Provincial Postdoctoral Science Foundation, as well as supported by Reform demonstration classes about teaching methods and assessment methods of "fundamentals of analog circuits" under grant 2017XJYJ-ZD06 
The work of Weijian Chen was supported by the integrated teaching of "circuit analysis" and "fundamentals of analog circuits" under grant 2015XJYZC004

The work of Jin Zhang was supported by comprehensive reform of undergraduate education in communications under grant 2015XJYZD001, as well as supported by professional certification as an opportunity to continuously improve the communications engineering professionals training under grant 2015XJYZD002.

The work of Quanyuan Feng was supported by the National Natural Science Foundation of China (NSFC) under Grant 61531016, supported by the project of Science and Technology Support Program of Sichuan Province under Grant 2015GZ0103, and in part by the Sichuan Provincial Science and Technology Important Projects under Grant 2017 GZ0110.

\section{REFERENCES}

[1] G. Qiu, and X. Luo, Circuit (Fourth Edition) [M], Beijing: Higher Education Press, June 1999.

[2] W. Zhang, Z. Tan, L. Li, and C. Fan, "Exploration of circuit teaching reform [J]," Journal of Electrical \& Electronic Education, vol. 30, no. 7, pp. 62-64, 2008.

[3] S. T. Karris, Electronic Devices and Amplifier Circuits with MATLAB Applications, Orchard Publications, 2005.

[4] T. L. Floyd and D. Buchla, Fundamentals of Analog Circuits, 2004.

[5] D. A. Neamen, Electronic Circuit Analysis \& Design, 2007.

[6] T. S. Bai and H. Ying, Analog Electronic Technology Foundation [M], Beijing: Higher Education Press, 2007.

[7] H. Kang and D. Chen, Fundamentals of Elecotronic Technology (Fourth Edition) [M], Beijing: Higher Education Press, 1999.

[8] J. Zhang and Y. Li, Based on Four Integration of Communication Personnel Training Model Reform and Practice [J], Education and Teaching Forum, 2016, 2, pp. 104-105.

Fan Hua was born in Ziyang, Sichuan Province, P.R. China, on Dec. 31, 1981. She received the Ph.D. degree from Tsinghua University, BeiJing, in 2013, from September 2013 to July 2016, she served as lecturer of the University of Electronic Science and Technology, since July 2016, served as an associate professor of the University of Electronic Science and Technology. As the project leader, she hosts two national nature Science funds, the Fundamental Research Funds for the Central Universities and China Postdoctoral Science Foundation, etc. She mainly engaged in analog chip design, "fundamentals of analog circuits" curriculum reform research. As the first author, she has published 8 SCI papers in IEEE Transactions on Circuits and Systems II and other international journals, and 10 EI papers, made presentations on international conference 6 times, as the first inventor she have applied for a US patent, a British patent, 15 Chinese patents, of which three have been authorized. In August 25, 2016, she won the second prize in the young teachers teaching competition in the southwest of China organized by the Ministry of Education; 2016 in December, she achieved the "outstanding young teacher growth plan" teaching excellence award; 2017 in April, won the first prize in the Young Teachers Teaching Competition in University of Electronic Science and Technology.

Chen Weijian is professor, chief teaching reform expert of "electronic circuit" course. In 1982 February graduated from Shanghai Jiaotong University undergraduate. 1982 to 1985 in the state-owned 432 plant in the design and development work. In April 1988, he graduated from Chongqing University. From 1988 to 1993, he was engaged in teaching and research work at the Department of Radio Technology, University of Electronic Science and Technology of China. From 1994 to 2002, he served as deputy director and deputy factory director and chief engineer of the factory. He was engaged in management and design and development work. Communication and Information Engineering College engaged in teaching and research work. Commitment to a number of graduate students and undergraduate course teaching work, a number of national key laboratory funds and production and research major scientific research work, a variety of products and engineering design and development work. Won the national teaching achievement award, the provincial science and technology progress award, the state-level new product development award and many schoollevel teaching achievement award. The current research directions: wireless and mobile communications, multimedia communications, communications, information security technology, cryptography theory and technology applications, communications networks and information systems security.

Zhang Jin is a lecturer, 1998.9-2004.8 Nanjing University of Science and Technology Master degree, School of Communication and Information Engineering, University of Electronic Science and Technology, chief of education, mainly engaged in teaching management, teaching research and reform to promote the implementation of the work. 2014 won the second prize in national teaching achievement, first prize Sichuan province teaching achievement, in 2016, won first prize by the school teaching achievement, in 2008 won the second prize in school teaching achievement. She conducts main research of 1 national quality engineering project, 2 Sichuan province teaching reform project, more than 10 school level teaching reform project, as the first author, she published 5 teaching and research papers. Access to advanced school workers, "three education" advanced individual, school excellent counselors, management of advanced individuals andso on. 